\title{
Analysing a Single Osteoblast Cell: Mechanotransduction Using Atomic Force Microscope Indention
}

Paula Maguire, James McGarry, Veronica Campbell, Brian O'Connell, Patrick Prendergast and Suzanne Jarvis.

Centre for Research on Adaptive Nanostructures and Nanodevices, University of Dublin, Trinity College, Dublin 2, Ireland.

Bone is a highly organized metabolically active tissue which is structurally tuned to its external environment. The morphology of bone can alter depending on its physical environment, responding to physical conditions such as mechanical stress. Mechanotransduction is a signaling process where an external mechanical signal is perpetuated into a controlled cascade of biological events. However, as of yet the initial sensor event in mechanotransduction has not been elucidated.

Many experimental systems have been developed to attempt to generate and investigate physical mechanical stimuli in vivo and in vitro. In this study an atomic force microscope (AFM) coupled to an Olympus fluorescence system was used to investigate mechanotransduction in a single MC3TCE1 osteoblast cell. AFM coupled to an Olympus fluorescence system has major advantages as a tool to investigate mechanotransduction. AFM allows precise application of very low forces while inducing minimal distribution to the cell. The fluorescence system allows study of cellular responses in real time in sync with the initiation of the indention process. This allows rapid signaling events such as increases in intracellular calcium levels and nitric oxide to be studied.

An increase in intracellular calcium and activation of nitric oxide are known secondary messengers in the transduction of stress inducing stimuli. This study monitors increases in intracellular calcium levels and nitric oxide after AFM indention (Fig. 1). A range of mechanical forces, at various frequencies, has been applied in order to establish a response threshold. Further, we have attempted to apply forces at various cellular locations to assess the importance of cellular location in the transduction of the mechanical stimulus. Cell to cell communication will also be examined. Understanding of the initial transduction of a mechanical signal will aid in finding the initial "mechanoreceptor" and aid in the understanding of the process of bone formation.

This research was supported by Science Foundation Ireland CSET Grant (03/CE3/M406). 

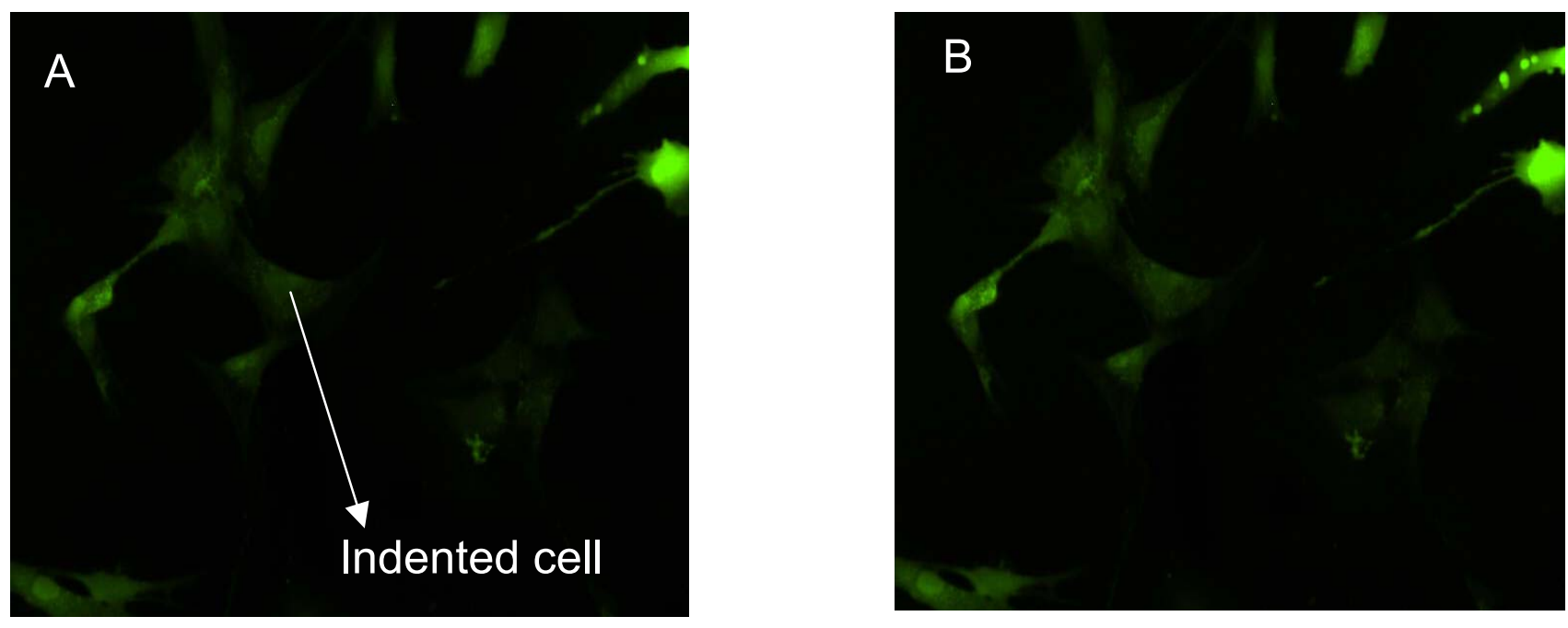

Figure 1. MC3T3-E1 osteoblast cells (x20) stained with 5 $\mathrm{M}$ DAF-FM. (A) Before AFM indention. Illustrates baseline fluorescence value (B) After AFM indention. An increase in green fluorescence in the indented cell indicates an increase in nitric oxide. 